\title{
Implantation of stentless aortic root prosthesis using an inversion technique
}

\author{
Niranjan Hiremath, MCh, FVES, FACS(Aus), ${ }^{a, b}$ and Gopal Bhatnagar, MD, ${ }^{a}$ Abu Dhabi, United Arab \\ Emirates, and Cleveland, Ohio
}

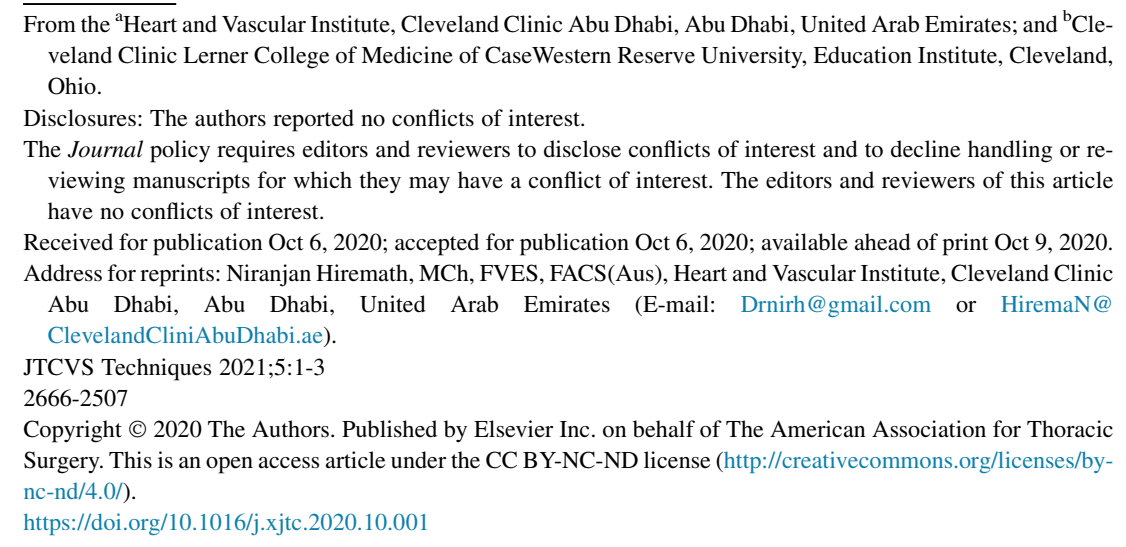

Infective endocarditis (IE) of the aortic valve is known to cause extensive destruction of the aortic annulus sometimes involving the aorta-mitral curtain. We present a technique of aortic xenograft implantation in a case of IE.

\section{Case Report}

A 19-year-old previously well male patient was referred to us from an outside facility, with a 10-day history of intermittent fever and progressive shortness of breath (New York Heart Association class III). His blood culture was positive for methicillin-susceptible Staphylococcus aureus, and he was administered broad-spectrum antibiotics. Findings of the transesophageal echocardiogram showed a Sievers type I bicuspid aortic valve with moderate aortic insufficiency and a $2-\mathrm{cm} \times 2-\mathrm{cm}$ vegetation attached on the ventricular side of the aortic leaflet, a $4-\mathrm{cm} \times 3-\mathrm{cm}$ vegetation attached to the A2 segment of mitral valve (MV) with perforation causing moderate mitral regurgitation, and an aortomitral pseudoaneurysm with positive Doppler flow and paradoxical expansion. Magnetic resonance imaging of the brain revealed multiple foci of septic emboli with an incidental finding of a small, localized subarachnoid hemorrhage and a $3-\mathrm{cm}$ aneurysm in the right pre-central sulcus. The patient had no neurologic manifestations and, after a multidisciplinary team discussion involving the neurologist, the patient was planned for urgent cardiac surgery (within 12 hours of presentation) based on class $1 \mathrm{~B}$ and class IIaB recommendations of AATS guidelines. ${ }^{1}$ To decrease the postoperative risk of anticoagulationrelated bleeding, patient was consented for a bioprosthetic valve. A consent for publication was also obtained.

\section{Technique}

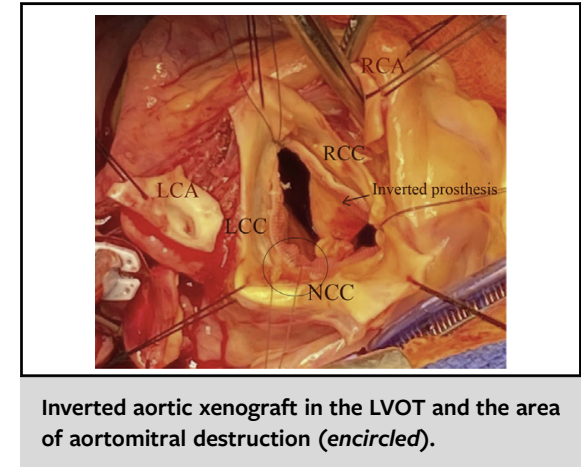

\section{CENTRAL MESSAGE \\ We describe a technique of im- plantation of aortic root pros- thesis using an inversion technique to enhance visualiza- tion and accurate suture place- ment in a case of infective endocarditis.}

See Commentaries on pages 4 and 6.

Cardiac exposure was obtained via sternotomy. The aorta and both cavae were cannulated, cardiopulmonary bypass was initiated, and cardioplegic arrest was achieved using del Nido cardioplegia. The aortic valve was excised through a transaortic approach and the aortomitral aneurysm was debrided. The MV was approached via left atriotomy, the anterior mitral leaflet vegetations were excised, and the perforation was repaired using a bovine pericardial patch (Figure 1, A). The left ventricular cavity and the aortomitral area was thoroughly irrigated with an antibiotic solution. A 25-mm Freestyle (Medtronic Inc, Minneapolis, Minn) porcine aortic root prosthesis was sized, carefully turned inside-out (Figure 1, $B$ ), and placed inside the left ventricular outflow tract in an inverted fashion (Figure 2) just below the aortic annulus, aligning the right coronary ostia of the prosthesis to the noncoronary sinus of the patient (Figure 1,C). The prosthesis depth was secured with 3 "commissural" 3-0 PROLENE sutures, which were then used to suture the prosthetic 

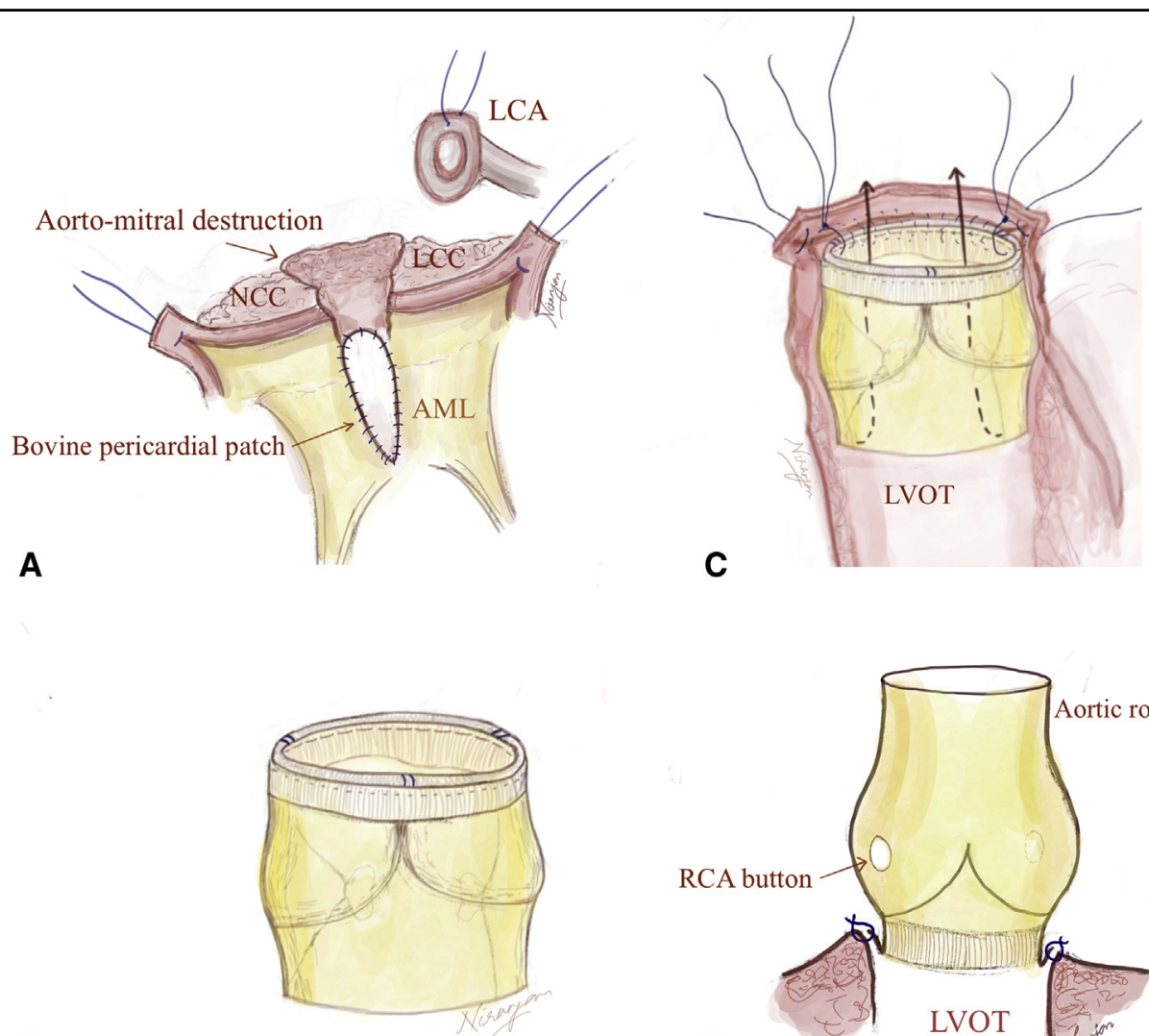

B

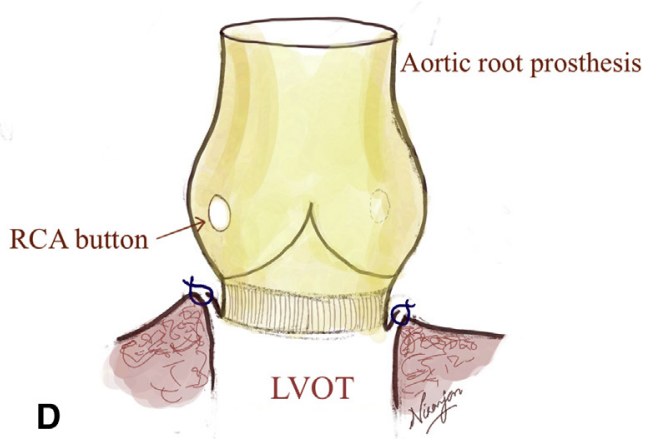

FIGURE 1. A, Repair of AML perforation using a bovine pericardial patch with the upper edge forming a part of the aortic annulus. Aortomitral discontinuity noted at the junction of LCC and NCC. B, Inverted prosthesis. C, Placed in the LVOT with the prosthesis suture rim just below the aortic annulus anchored with PROLENE sutures. D, Root prosthesis everted back to the external position with RCA and LCA buttons prepared for implantation. $L C A$, Left coronary artery; $N C C$, noncoronary cusp; $L C C$, left coronary cusp; $A M L$, anterior mitral leaflet; $L V O T$, left ventricular outflow tract; $R C A$, right coronary artery.

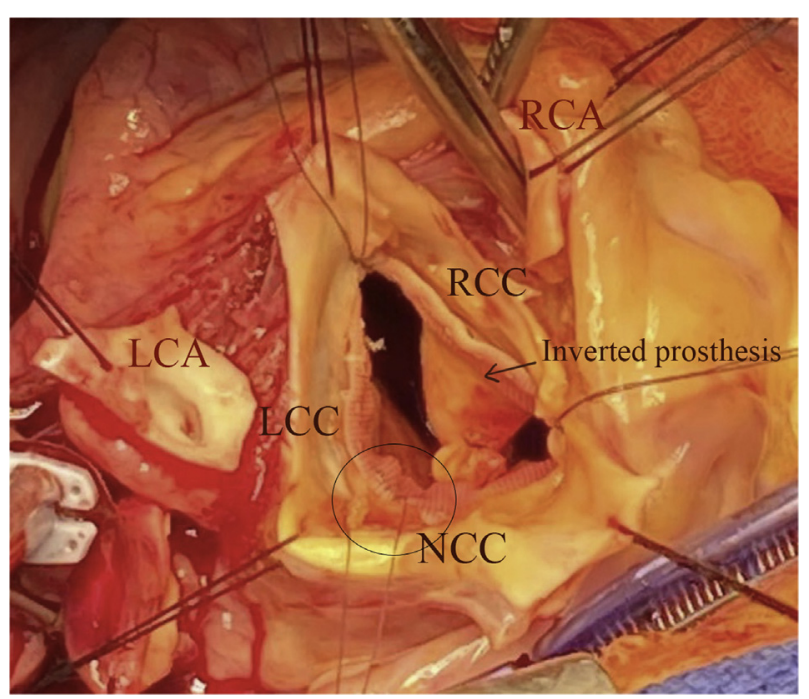

FIGURE 2. Inverted Freestyle prosthesis in the LVOT as seen from above with the encircled area showing aortomitral discontinuity above the prosthetic rim. $R C A$, Right coronary artery; $R C C$, right coronary cusp; $L C A$, left coronary artery; $L C C$, left coronary cusp; $N C C$, noncoronary cusp. rim to the aortic annulus in a continuous fashion. The annulus at the noncoronary cusp was reconstructed with a bovine pericardial strip, thus obliterating the aortomitral defect and integrated into the prosthetic suture line. The prosthesis was then everted back to normal orientation (Figure 1,D) and both coronary ostia were reimplanted using 5-0 PROLENE sutures. Finally, the distal graft to ascending aorta anastomosis was completed. The intraoperative TEE demonstrated normal functioning aortic root prosthesis, a mild central leak in the MV, and successful repair of aortomitral defect. The patient was separated from cardiopulmonary bypass successfully with no rhythm abnormalities.

The patient had an uneventful postoperative stay in the intensive care unit. The microbiology result of the excised tissue was negative; however, the patient received 6 weeks of intravenous flucloxacillin in the hospital based on the initial positive blood culture. He was discharged after completion of antibiotic course and had no neurologic deficits at the time of discharge. He was completely well at 6 weeks follow-up. 


\section{DISCUSSION}

Xenografts are readily available (compared with homograft) tissue valves and known for their superior outcomes in surgical treatment of IE. ${ }^{2,3}$ Many techniques of stentless allograft implantation within the aortic $\operatorname{root}^{4}$ and inversion technique of older generation valves ${ }^{5}$ have been described that rely on the presence of native aortic tissue for anchorage. In our technique, an entire porcine root prosthesis can be inverted and implanted to reconstruct the aortic root. We have found that by inverting the prosthesis, a lower level of implantation in the left ventricular outflow tract can be achieved, especially in situations involving loss of annular tissue. This enhances visualization of the suture line, which is otherwise limited by stiffness of the prosthetic sinuses and restricted suturing space as encountered in redo surgeries, small aortic root, and extensive aortic root destruction. This technique enables precise suture placement, which is important to not cause mal-alignment of the prosthetic leaflets and externalizing any abscess cavity also ensuring that no Dacron material is exposed to the circulation. Use of the stentless root prosthesis gives excellent outflow hemodynamics and reduces stress on a fragile outflow tract, obviating the need for extensive reconstruction, which can compromise the annular diameter.

\section{References}

1. AATS Surgical Treatment of Infective Endocarditis Consensus Guidelines Writing Committee Chairs, Pettersson GB, Coselli JS, Writing Committee, Hussain ST, Griffin B, et al. 2016 the American Association for Thoracic Surgery (AATS) consensus guidelines: surgical treatment of infective endocarditis: executive summary. J Thorac Cardiovasc Surg. 2017;153:1241-58.e29.

2. Heinz A, Dumfarth J, Ruttmann-Ulmer E, Grimm M, Müller LC. Freestyle roo replacement for complex destructive aortic valve endocarditis. J Thorac Cardiovasc Surg. 2014;147:1265-70.

3. Schneider AW, Hazekamp MG, Versteegh MI, Bruggemans EF, Holman ER, Klautz RJ, et al. Stentless bioprostheses: a versatile and durable solution in extensive aortic valve endocarditis. Eur J Cardiothorac Surg. 2016;49: 1699-704.

4. Westaby S, Amarasena N, Ormerod O, Amarasena GA, Pillai R. Aortic valve replacement with the freestyle stentless xenograft. Ann Thorac Surg. 1995;60(2 suppl):S422-7.

5. O'Brien MF, McGiffin DC, Stafford EG. Allograft aortic valve implantation: techniques for all types of aortic valve and root pathology. Ann Thorac Surg. 1989; 48: 600-9. 